\title{
Impact of radiotherapy for localized prostate cancer on bladder function as demonstrated on urodynamics study: A systematic review
}

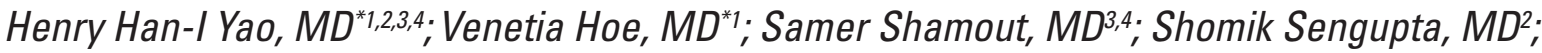 \\ Helen E. O'Connell, MD'; Kevin V. Carlson, MD ${ }^{3,4}$; Richard J. Baverstock, MD ${ }^{3,4}$
}

'Department of Urology, Western Health, Melbourne, Australia; 2 Eastern Health Clinical School, Eastern Health, Melbourne, Australia; ${ }^{3}$ vesia [Alberta Bladder Centre], Calgary, AB, Canada; ${ }^{4}$ Department of Surgery, University of Calgary, Calgary, AB, Canada

*Equal contributors

Cite as: Yao H.H-I, Hoe V, Shamout S, et al. Impact of radiotherapy for localized prostate cancer on bladder function as demonstrated on urodynamics study: A systematic review. Can Urol Assoc J 2021;15(12):E664-71. http://dx.doi.org/10.5489/cuaj.7265

Published online June 22, 2021

\section{Abstract}

Introduction: This study aimed to describe the effects of bladder function following radiotherapy for localized prostate cancer by performing a systematic review on studies reporting on urodynamic findings after radiotherapy.

Methods: This systematic review was conducted in accordance with PRISMA guidelines. The review protocol was registered at PROSPERO (CRD42021229037). A systematic search was conducted using PubMed, Cochrane Library, Scopus, and OVID Embase. Studies were included if they involved men who underwent urodynamic studies following radiotherapy for localized prostate cancer. A total of 798 articles were screened and five articles included. A qualitative analysis was performed.

Results: Bladder compliance appears to be impaired following radiotherapy, especially with longer followup. Impaired bladder compliance was reported in $18.8-62.5 \%$ of patients following radiotherapy. Bladder capacity was found to be statistically significantly lower following radiotherapy compared to pre-radiotherapy, and when compared with patients who did not undergo pelvic radiotherapy. Bladder outlet obstruction (BOO) persists post-radiotherapy in most patients at three and 18 months post-radiotherapy. De novo detrusor overactivity (DO) of $13.3 \%$ has been reported at 18 months post-radiotherapy. This review is limited by the absence of level I/II studies.

Conclusions: Radiotherapy for localized prostate cancer results in decreased bladder compliance and capacity demonstrated on urodynamic studies. Resolution of BOO appears less likely in comparison to series on radical prostatectomy. De novo DO may develop following radiotherapy, especially with longer followup. With only low level of evidence studies available at present, further high-quality, prospective studies are important to elucidate the impact of radiotherapy on bladder and urethral function.

\section{Introduction}

Prostate cancer is the second most diagnosed cancer in men. ${ }^{1}$ Curative management options for localized prostate cancer include radical prostatectomy (RP) and radiotherapy. Conventional radiotherapy techniques, such as external beam radiotherapy and low-dose brachytherapy, have demonstrated similar efficacy to RP with regard to oncological outcomes. ${ }^{2}$ Both RP and radiotherapy as curative treatment provide patients with a favorable long-term overall and cancer-specific survival rate. ${ }^{3}$ With a prolonged survival following curative treatment, achieving optimal functional and quality-of-life (QoL) outcomes are important. The trifecta of cancer control, urinary continence, and erectile function are heavily studied, ${ }^{4}$ but bladder function less frequently so. ${ }^{5}$

Lower urinary tract symptoms (LUTS) are a well-known side effect of radiotherapy for prostate cancer, due to genitourinary toxicity. ${ }^{5}$ There is a paucity of data on bladder function following radiotherapy for prostate cancer. The most objective method to determine changes in bladder function is with urodynamic studies.

This study aims to determine the effects of bladder function following radiotherapy treatment of prostate cancer by performing a systematic review on urodynamic findings before and after radiotherapy. The results of this study will assist clinicians in the counselling of patients regarding the effect of radiotherapy treatment of prostate cancer on bladder function outcomes post-radiotherapy.

\section{Methods}

This systematic review was conducted in accordance with the PRISMA guideline. The review protocol was registered at PROSPERO (https://www.crd.york.ac.uk/prospero/): registration number (CRD42021229037).

The study cohort include patients with localized prostate cancer treated with radiotherapy. This systematic review 
included patients who were treated both in the primary setting, as well as adjuvant or salvage setting following prostatectomy. Studies were eligible to be included if there were urodynamic studies performed following radiotherapy to assess the impact of radiotherapy on bladder function. Articles were excluded if they were non-English articles, non-full-text articles, review articles, and other article types with no original data.

The primary outcome assessed in this study is the rate of urodynamic changes (detrusor overactivity [DO], bladder compliance, maximum cystometric capacity, bladder contractility, and bladder outlet obstruction [BOO]). As there is significant heterogeneity of the underlying patient population across the study, and incomplete reporting of each of the outcomes for different studies, a quantitative analysis could not be performed. A descriptive, qualitative analysis is reported.

A systematic search was conducted independently by two investigators using PubMed, Cochrane library, Scopus, and OVID Embase on October 16, 2020. The complete search protocol, with inclusion and exclusion criteria, are listed in Fig. 1.

Abstracts were screened independently by two investigators to identify articles to be included. Full-text articles were reviewed if eligibility to be included in the review was not able to be determined from the title and abstract. Discrepancies of assessment between investigators were resolved following a discussion to reach a consensus. Risk of bias assessments were performed individually by two investigators using the National Heart, Lung, and Blood Institute Study Quality Assessment Tools. ${ }^{6}$ Data were collected into an electronic data collection form and included baseline demographics data, method of radiotherapy, dose and fraction of radiotherapy, baseline disease characteristics, patient-reported outcome measures (PROMs) data at baseline and followup, and urodynamics data at baseline and following radiotherapy.

\section{Results}

A total of 798 articles were screened to assess for eligibility. After removing 75 duplicates, 723 articles were screened against title and abstract and 19 articles assessed for full-text eligibility. Five of the 19 articles were eligible to be included in the final review. ${ }^{7-11}$ Reasons for exclusion are listed in Fig. 1. Two of the articles came from the same cohort of patients with different lengths of followup, and therefore, were analyzed together as one study. The final number of studies included was four: two retrospective cohort studies, one retrospective pre-post study published in two articles with different followup times, and one case series. Only low-level evidence (3 or 4) studies were found in the literature for this systematic review. On risk of bias assessment, two articles were found to have a low risk of bias and three articles were found to have at least a moderate risk of bias for the type of study conducted (Supplementary Table 1).

\section{Baseline demographics}

One of the four studies included both prostate cancer and colorectal patients, with most of the patients having radiotherapy for prostate cancer $(n=58 / 99) .{ }^{10}$ This study also had a control group of non-radiotherapy patients for comparison. ${ }^{10}$ One study involved patients with primary curative radiotherapy only. ${ }^{7,8}$ Another study included patients following salvage radiotherapy post-RP. ${ }^{9}$ The final study included patients following adjuvant radiotherapy and compared with patients who underwent RP alone as control. ${ }^{11}$ The number of prostate cancer radiotherapy patients in each study ranged from 10-58 (Table 1). The sum total of prostate cancer radiotherapy patients included in this systematic review was 100 . The median age of patients at time of radiotherapy ranged from 64.9-72.0 years. Prostate volume was not reported in all studies. Prostate cancer disease characteristic were reported in only two articles that belonged to the same study (Table 1).

External beam radiotherapy was used in all studies. Two studies used 3D conformal therapy, ${ }^{9,10}$ one study used fourfield box technique, ${ }^{7,8}$ and one study included patients with both techniques. ${ }^{11}$ The dose of radiation ranged from 59-72 Gy. ${ }^{7-9,11}$ One study included colorectal cancer patients and reported the radiation dose to range from 24-78 Gy..$^{10}$ The authors of that study did not specify the dose used in prostate cancer but it is likely that the dose would be in the higher end of the reported range when used to treat prostate cancer, in keeping with standard practice. Only one study reported the dose of bladder exposure to the radiation with a median of $81.7 \mathrm{ml}$ of bladder within $80 \%$ of radiation isodose and median of $134.8 \mathrm{ml}$ within $50 \%$ of radiation isodose., The bladder was filled during this study in order to reduce the amount of bladder exposure.,

\section{Symptoms and PROMs}

Only one study reported changes to symptoms and PROMs compared with baseline (Table 2). ${ }^{7,8}$ In this study, the baseline median International Prostate Symptoms Score (IPSS) was 7 (range 1-22), the median IPSS QoL score was 1.5 (range $0-5$ ), and the baseline median urinary frequency per 24 hours was 8.5 (range 4-14). ${ }^{7,8}$ Urgency was reported in $62.5 \%$ of men and urge urinary incontinence in $25 \%$ of men prior to radiotherapy. ${ }^{7,8}$ Median urinary frequency, IPSS, and IPSS QoL scores were not statistically significantly different at three and 18 months compared with baseline. ${ }^{7,8}$ Overall, the percentage of men with urgency symptoms was worse at three months following radiotherapy, as $31.3 \%$ of men developed de novo urgency, $43.8 \%$ of men had persistent urgency, and only $18.8 \%$ had de novo resolution of urgency symptoms. ${ }^{8}$ Similarly, the percentage of men with urge urinary incontinence was higher at three months, with $18.8 \%$ developing de novo urge urinary incontinence, $25 \%$ 

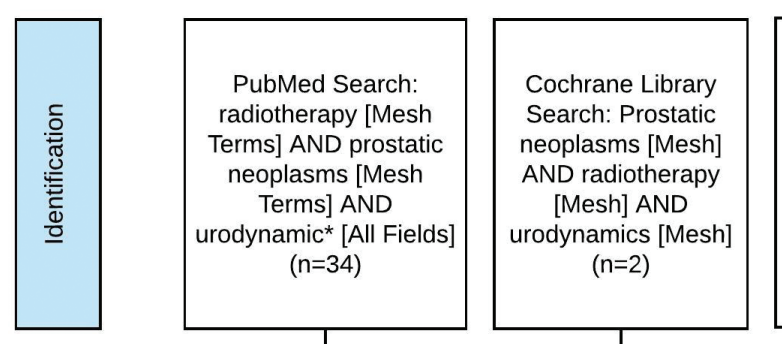
\begin{tabular}{|} 
OVID Embase Search: \\
(radiotherapy [Subject Heading \\
OR radiotherapy [Keyword]) \\
AND (prostate cancer [Subject \\
Heading] OR prostate cancer \\
[Keyword]) AND (urodynamics
\end{tabular}

[Subject Heading] OR urodynamics [Keyword]) $(n=96)$
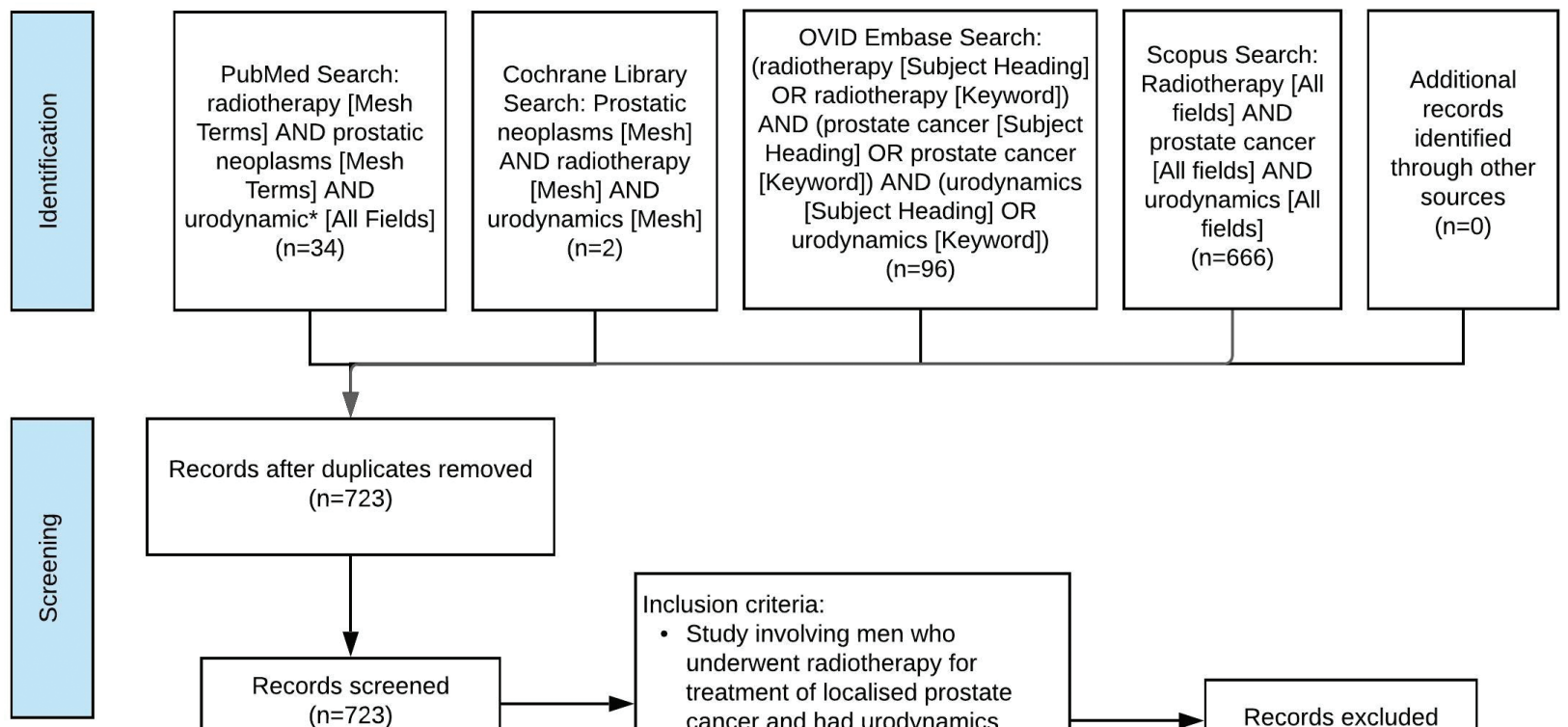

Records after duplicates removed $(n=723)$
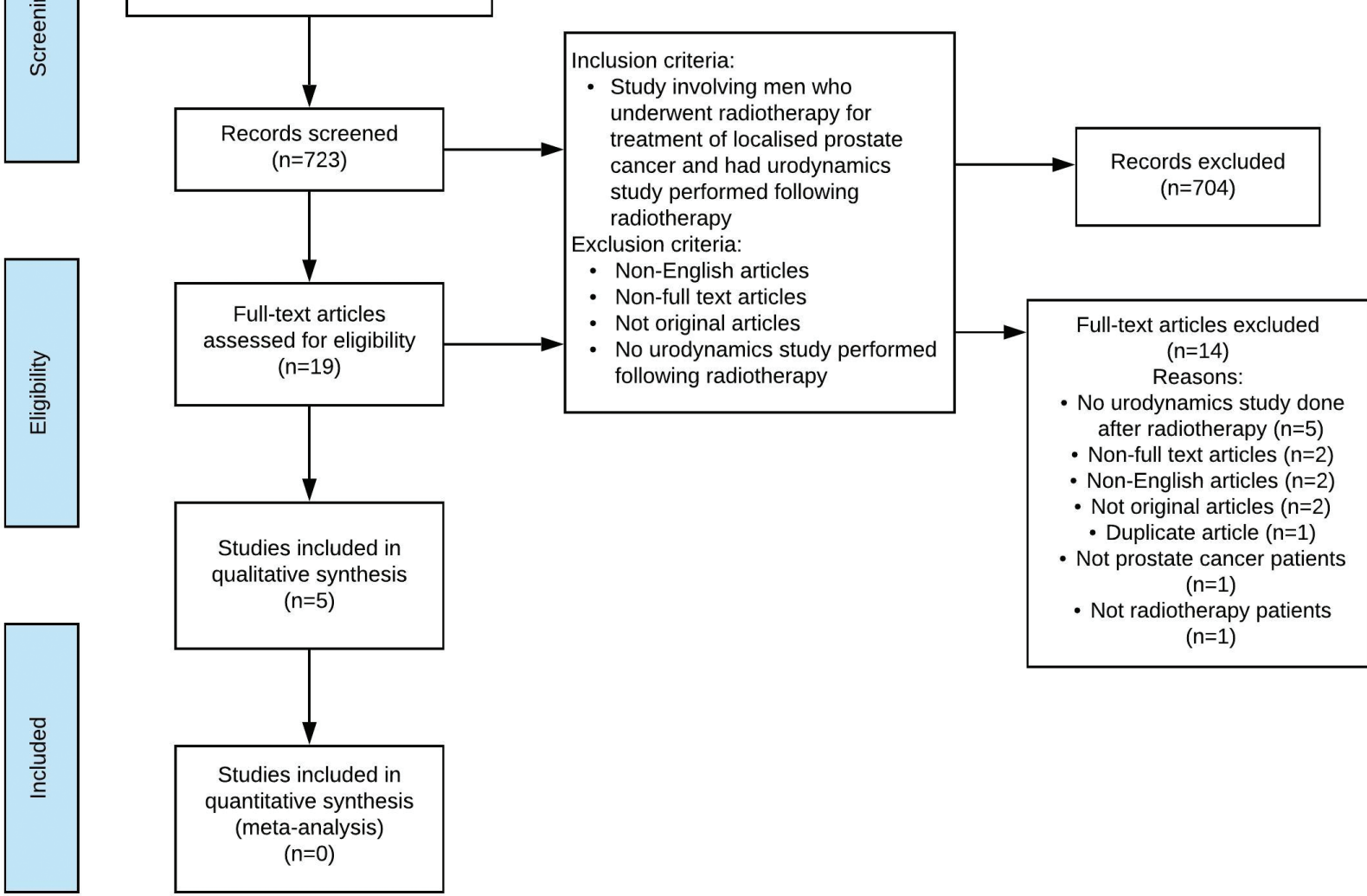

usion criteria underwent radiotherapy for treatment of localised prostate cancer and had urodynamics study performed following

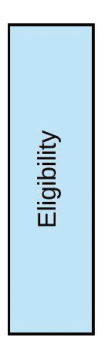

xclusion criteria:

Non-English articles

Non-full text articles

urodynamics study performed fllowing radiotherapy

$(n=14)$

No urodynamics study done

Non-English articles $(n=2)$

original articles $(n=2)$

Not prostate cancer patients

- Not radiotherapy patients $(n=1)$

Fig. 1. Results of search strategy using PubMed, Scopus, EMBASE, and Cochrane.

of men experiencing persistent urge urinary incontinence, and no de novo resolution following radiotherapy. ${ }^{8}$ These rates remain largely unchanged at 18 months compared with three months post-radiotherapy. ${ }^{7.8}$ Only one other study reported PROMs outcome and found $62.5 \%$ of men to have moderate or severe Danish Prostatic Symptom Score (DANPSS) at median of 7.7 years following salvage radiotherapy. ${ }^{9}$

\section{Bladder outlet obstruction}

Changes to urodynamic BOO were reported by only one study. ${ }^{7,8}$ The rate of $\mathrm{BOO}$ demonstrated on urodynamics at baseline was $81.3 \% .{ }^{7.8} \mathrm{BOO}$ was largely persistent, with $75 \%$ of patients still experiencing $\mathrm{BOO}$ at three months and $60 \%$ of men at 18 months. ${ }^{78}$ Twenty percent of men experienced de novo resolution of $\mathrm{BOO}$ following radiotherapy at 18 months. ${ }^{7}$ A small percentage $(6.3 \%$ ) of men experienced de novo $\mathrm{BOO}$ at three months but this was resolved by 18 months. ${ }^{7,8}$ The maximum flow rate (Qmax) and postvoid residual (PVR) did not appear to be impacted following radiotherapy at three and 18 months. ${ }^{7,8}$. Ervandian et al did not compare BOO rates with baseline and reported a significant rate of $\mathrm{BOO}$ of $43.8 \%$ at a median of 7.7 years following salvage radiotherapy, with an additional $25 \%$ of 
Table 1. Baseline disease and treatment characteristics of studies examining the impact of radiotherapy for prostate cancer on urodynamics findings

\begin{tabular}{|c|c|c|c|c|c|c|c|c|c|c|}
\hline Author & Study type & Number & Age & $\begin{array}{c}\text { Initial PSA } \\
\text { (ng/mL) }\end{array}$ & $\begin{array}{l}\text { Biopsy } \\
\text { grade }\end{array}$ & $\begin{array}{c}\text { Clinical T } \\
\text { Stage }\end{array}$ & $\begin{array}{l}\text { Method } \\
\text { of RT }\end{array}$ & $\begin{array}{c}\text { Technique } \\
\text { details }\end{array}$ & $\begin{array}{c}\text { Dose/ } \\
\text { fractions }\end{array}$ & $\begin{array}{l}\text { Bladder within } \\
\text { radiation field }\end{array}$ \\
\hline $\begin{array}{l}\text { Ervandian } \\
\text { et al, } \\
2018^{9}\end{array}$ & Case series & $\begin{array}{c}\text { RRP + } \\
\text { salvage } \\
\text { RT ( } n=15) ; \\
\text { RARP + } \\
\text { salvage RT } \\
\quad(n=1)\end{array}$ & $\begin{array}{c}\text { Median } \\
\text { age at } \\
\text { RRP 62.5 } \\
\text { (52.8- } \\
\text { 72.1) and } \\
\text { median } \\
\text { age at } \\
\text { RT 64.9 } \\
\text { (56.2- } \\
73.4)\end{array}$ & $\begin{array}{c}\text { Pre-RRP } \\
\text { PSA: }<10 \text { in } \\
3,10-20 \text { in } 9 \text {, } \\
>20 \text { in } 4 . \text { Pre- } \\
\text { RT PSA: } 0.2 \\
\text { in 1, } 0.2-0.5 \\
\text { in 2, } \geq 0.5 \text { in } \\
\text { 11, unknown } \\
\text { in } 2\end{array}$ & $\mathrm{~N} / \mathrm{A}$ & $\mathrm{N} / \mathrm{A}$ & EBRT & $\begin{array}{c}\text { 3D } \\
\text { conformal } \\
\text { therapy }\end{array}$ & $\begin{array}{c}68 \mathrm{~Gy} / 34 \\
\text { fractions } \\
\text { (except } \\
1 \text { patient } \\
\text { had } 72 \\
\text { Gy/36 } \\
\text { fractions) }\end{array}$ & $\mathrm{N} / \mathrm{A}$ \\
\hline $\begin{array}{l}\text { Mendez- } \\
\text { Rubio et } \\
\text { al, } 2015^{10}\end{array}$ & $\begin{array}{l}\text { Retrospective } \\
\text { cohort study }\end{array}$ & $\begin{array}{c}\text { Total } \\
\text { number } \\
\text { (n=99); } \\
\text { primary } \\
\text { RT (n=49); } \\
\text { adjuvant } \\
\text { RT ( } \mathrm{n}=50) ; \\
\text { prostate } \\
\text { cancer } \\
\text { (n=58); } \\
\text { colorectal } \\
\text { cancer } \\
\text { (n=41) }\end{array}$ & $\begin{array}{l}\text { Mean } \\
69 \pm 8.5\end{array}$ & $\mathrm{~N} / \mathrm{A}$ & $\mathrm{N} / \mathrm{A}$ & N/A & EBRT & $\begin{array}{c}\text { 3D } \\
\text { conformal } \\
\text { therapy }\end{array}$ & $\begin{array}{c}\text { 24-78 } \\
\text { Gy/12-39 } \\
\text { fractions } \\
\text { at daily } \\
\text { dose of } \\
2 \text { Gy and } \\
\text { between } \\
2-8 \\
\text { weeks of } \\
\text { treatment }\end{array}$ & $\mathrm{N} / \mathrm{A}$ \\
\hline $\begin{array}{l}\text { Do et al, } \\
2002^{8} \text { and } \\
\text { Choo et } \\
\text { al, } 2002^{7 *}\end{array}$ & $\begin{array}{l}\text { Pre-post } \\
\text { study }\end{array}$ & $\begin{array}{c}16 / 17 \\
\text { completed } \\
\text { the study }\end{array}$ & $\begin{array}{c}\text { Median } \\
72 \text { (range } \\
56-77 \text { ) }\end{array}$ & $\begin{array}{c}\text { Median } \\
8.55 \text { (range } \\
1.1-49.7 \text { ) }\end{array}$ & $\begin{array}{l}\text { Median } \\
7(6-8)\end{array}$ & $\begin{array}{c}\text { T1c }(n=3) ; \\
\text { T2 }(n=12) ; \\
\text { T3 }(n=1)\end{array}$ & EBRT & $\begin{array}{c}\text { Four- } \\
\text { field box } \\
\text { technique } \\
\text { and high } \\
\text { energy } \\
\text { photons } \\
\text { (18 or } 23 \\
\text { MV) }\end{array}$ & $\begin{array}{c}66 \mathrm{~Gy} / 33 \\
\text { fractions } \\
\text { to } 70 \\
\text { Gy/35 } \\
\text { fractions }\end{array}$ & $\begin{array}{c}\text { Within } 100 \% \\
\text { of radiation } \\
\text { isodose=median } \\
0.9(0-19.5) \% \\
\text { and median } \\
4.1(0-36.3) \\
\text { mL; within } 80 \% \\
\text { of radiation } \\
\text { isodose=median } \\
30.6 \% \text { and } \\
\text { median } 81.7 \\
\text { (43.4-151) mL; } \\
\text { within } 50 \% \text { of } \\
\text { radiation isodose } \\
\text { =median } 60.6 \% \\
\text { and median } 134.8 \\
\text { (72-500) mL }\end{array}$ \\
\hline $\begin{array}{l}\text { Presti et } \\
\text { al, } 1996^{11}\end{array}$ & $\begin{array}{l}\text { Prospective } \\
\text { cohort study }\end{array}$ & $\begin{array}{c}\text { Adjuvant } \\
\text { RT after } \\
\text { RRP } \\
(n=10) ; \\
\text { RRP only } \\
(n=13)\end{array}$ & $\mathrm{N} / \mathrm{A}$ & N/A & $\mathrm{N} / \mathrm{A}$ & $\mathrm{N} / \mathrm{A}$ & EBRT & $\begin{array}{c}\text { 3D } \\
\text { conformal } \\
\text { therapy } \\
(\mathrm{n}=8) ; \\
\text { 4-field } \\
\text { standard } \\
\text { RT }(\mathrm{n}=2)\end{array}$ & $\begin{array}{c}\text { Mean } \\
6328 \text { cGy } \\
\text { (range } \\
5940- \\
6500 \text { cGy) }\end{array}$ & $\mathrm{N} / \mathrm{A}$ \\
\hline
\end{tabular}

*These two studies were from the same population with different lengths of followup. EBRT: external beam radiation therapy; MV: megavolts; PSA: prostate-specific antigen; RARP: robotassisted radical prostatectomy; RRP: retropubic radical prostatectomy; RT: radiotherapy.

men unable to void with the presence of a urodynamics catheter. ${ }^{9}$ Similarly, the reported mean Qmax was poor at $9.6-11.4 \mathrm{ml} / \mathrm{s}^{9}$

\section{Detrusor overactivity}

Changes to the frequency of urodynamic DO were reported by only one study. ${ }^{7,8}$ The rate of DO at baseline was reported to be high at $56.3 \%$, in keeping with the significant symptoms of urgency and urge urinary incontinence reported by the same cohort of patients. ${ }^{7,8}$ This is in the setting of a baseline $\mathrm{BOO}$ of $81.3 \%$, suggesting that concurrent benign prostate hyperplasia $(\mathrm{BPH})$ is likely to play a role in the secondary DO. ${ }^{7,8}$ Overall, the rate of DO remained unchanged following radiotherapy at three months, and slightly worse at $60 \%$ at 18 months, with $13.3 \%$ of men having developed 
Yao et al

\begin{tabular}{|c|c|c|c|c|c|c|c|c|}
\hline Author & $\begin{array}{l}\text { Patient } \\
\text { selection }\end{array}$ & $\begin{array}{l}\text { Changes in overactive } \\
\text { bladder symptoms }\end{array}$ & $\begin{array}{l}\text { Changes in } \\
\text { PROMs }\end{array}$ & $\begin{array}{l}\text { Timing of } \\
\text { UDS }\end{array}$ & $\begin{array}{l}\text { Bladder } \\
\text { compliance }\end{array}$ & $\begin{array}{l}\text { Detrusor } \\
\text { overactivity }\end{array}$ & $\begin{array}{l}\text { Bladder } \\
\text { capacity }\end{array}$ & $\begin{array}{c}\text { Bladder } \\
\text { outlet } \\
\text { obstruction }\end{array}$ \\
\hline $\begin{array}{l}\text { Ervandian } \\
\text { et al, } \\
2018^{9}\end{array}$ & $\begin{array}{l}89 \text { patients } \\
\text { SRT patients } \\
\text { eligible; } 52 \\
\text { invited for } \\
\text { study; } 16 \\
\text { consented for } \\
\text { per-protocol } \\
\text { urodynamics }\end{array}$ & $\mathrm{N} / \mathrm{A}$ & $\mathrm{N} / \mathrm{A}$ & $\begin{array}{l}\text { Median } \\
\text { time } \\
\text { from } \\
\text { SRT=7.7 } \\
\text { years } \\
\text { (range } \\
5.8-10 \text { ) }\end{array}$ & $\begin{array}{c}\text { Low in } 10 \\
(62.5 \%) \\
\text { (defined as } \\
<30-40 \mathrm{ml} / \mathrm{cm} \\
\left.\mathrm{H}_{2} \mathrm{O}\right)\end{array}$ & $\begin{array}{c}\text { Detrusor } \\
\text { overactivity= } \\
6(37.5 \%)\end{array}$ & $\begin{array}{c}297.8 \pm 28.3 \\
\mathrm{~mL}\end{array}$ & $\begin{array}{c}7(43.8 \%) \\
\text { with } 4 \text { unable } \\
\text { to void }(25 \%)\end{array}$ \\
\hline $\begin{array}{l}\text { Mendez- } \\
\text { Rubio et } \\
\text { al, } 2015^{10}\end{array}$ & $\begin{array}{l}\text { Not stated as } \\
\text { per-protocol } \\
\text { urodynamics } \\
\text { or } \\
\text { urodynamics } \\
\text { performed for } \\
\text { symptoms }\end{array}$ & $\mathrm{N} / \mathrm{A}$ & $\mathrm{N} / \mathrm{A}$ & $\begin{array}{l}\text { Mean } \\
4.7 \text { years } \\
\text { (SD } 4.07 \\
\text { years) }\end{array}$ & $\begin{array}{l}\text { Rates not } \\
\text { reported; pelvic } \\
\text { radiotherapy } \\
\text { shown to be } \\
\text { a predictor } \\
\text { of decreased } \\
\text { compliance on } \\
\text { univariate and } \\
\text { multivariate } \\
\text { analysis }\end{array}$ & $\begin{array}{l}\text { Rates not } \\
\text { reported; } \\
\text { pelvic } \\
\text { radiotherapy } \\
\text { not found } \\
\text { to be a } \\
\text { predictor } \\
\text { of detrusor } \\
\text { overactivity }\end{array}$ & $\begin{array}{c}175 \pm 105.4 \\
\text { (c.f. } \\
236 \pm 128.0 \\
\text { in control } \\
\text { group* } \\
p=0.000 \text { ) }\end{array}$ & $\mathrm{N} / \mathrm{A}$ \\
\hline $\begin{array}{l}\text { Do et al, } \\
2002^{8} \text { and } \\
\text { Choo et } \\
\text { al, } 2002^{\text {7\# }}\end{array}$ & $\begin{array}{l}\text { Per-protocol } \\
\text { urodynamics } \\
\text { for all eligible } \\
\text { patients who } \\
\text { consented }\end{array}$ & 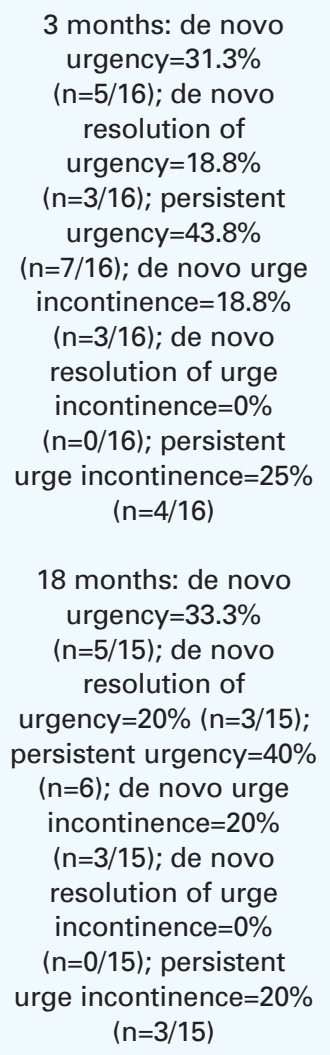 & $\begin{array}{c}3 \\
\text { months=mean } \\
\text { (SEM) change } \\
\text { for urinary } \\
\text { frequency= } \\
1.56(1.04) ; \\
\text { for IPSS= } \\
1.38(1.81) ; \\
\text { for QoL= } \\
0.06(0.39) ; \\
\text { statistically } \\
\text { not different } \\
\text { from baseline } \\
\\
18 \text { months= } \\
\text { mean (SEM) } \\
\text { change } \\
\text { for urinary } \\
\text { frequency= } \\
0.67 \text { (0.81); } \\
\text { for IPSS=0.4 } \\
\text { (1.63); for } \\
\text { QoL=0 (0.29); } \\
\text { statistically } \\
\text { not different } \\
\text { from baseline }\end{array}$ & $\begin{array}{l}3 \text { months } \\
\text { and } 18 \\
\text { months }\end{array}$ & $\begin{array}{c}3 \text { months: } \\
\text { de novo } \\
\text { decreased } \\
\text { compliance= } \\
18.8 \% \text { ( } n=3) ; \\
\text { de novo } \\
\text { resolution= } \\
12.5 \% \text { ( } n=2) \\
18 \text { months: } \\
\text { de novo } \\
\text { decreased } \\
\text { compliance= } \\
26.7 \% \text { ( } n=4 / 15) ; \\
\text { de novo } \\
\text { resolution= } \\
6.7 \%(n=1 / 15) ; \\
\text { persistent } \\
\text { decreased } \\
\text { compliance= } \\
6.7 \%(n=1 / 15)\end{array}$ & $\begin{array}{c}3 \text { months: } \\
\text { de novo } \\
\text { DO = 12.5\% } \\
(n=2) ; \\
\text { de novo } \\
\text { resolution= } \\
12.5 \% \\
\text { (n=2); DO } \\
\text { persistent }= \\
43.8 \% \text { ( } n=7) \\
\\
18 \text { months: } \\
\text { de novo } \\
\text { DO = 13.3\% } \\
\text { (n=2/15); } \\
\text { de novo } \\
\text { resolution= } \\
6.3 \% \\
\text { (n=1/15); DO } \\
\text { persistent= } \\
46.7 \% \\
(n=7 / 15)\end{array}$ & $\begin{array}{c}3 \text { months: } \\
\text { supine } \\
\text { mean (SEM) } \\
\text { change is } \\
-70 \mathrm{~mL}(29) \text {, } \\
\mathrm{p}=0.028 ; \\
\text { upright } \\
\text { mean (SEM) } \\
\text { change is } \\
-21 \mathrm{~mL}(29) \text {, } \\
\mathrm{p}=0.49 \\
18 \text { months: } \\
\text { supine } \\
\text { mean (SEM) } \\
\text { change } \\
\text { is }-100 \\
\text { mL ( } 20) \text {, } \\
\mathrm{p}=0.0002 ; \\
\text { upright } \\
\text { mean (SEM) } \\
\text { change is } \\
-54 \mathrm{~mL}(23) \text {, } \\
\mathrm{p}=0.035\end{array}$ & $\begin{array}{c}3 \text { months: } \\
\text { de novo } \\
\text { BOO=6.3\% } \\
(n=1) ; \\
\text { de novo } \\
\text { resolution= } \\
12.5 \% \\
(n=2) ; \text { BOO } \\
\text { persistent= } \\
68.8 \% \text { ( } n=11) \\
18 \text { months: } \\
\text { de novo } \\
\text { BOO=0\% } \\
\text { ( } n=0 / 15) ; \\
\text { de novo } \\
\text { resolution= } \\
20 \%(n=3 / 15) ; \\
\text { BOO } \\
\text { persistent= } \\
60 \%(n=9 / 15)\end{array}$ \\
\hline $\begin{array}{l}\text { Presti et } \\
\text { al, 1996 }\end{array}$ & $\begin{array}{l}\text { Not stated as } \\
\text { per-protocol } \\
\text { urodynamics } \\
\text { or } \\
\text { urodynamics } \\
\text { performed for } \\
\text { symptoms }\end{array}$ & $\mathrm{N} / \mathrm{A}$ & $\mathrm{N} / \mathrm{A}$ & $\begin{array}{c}\text { 3-22 } \\
\text { months } \\
\text { following } \\
\text { adjuvant } \\
\text { RT }\end{array}$ & $\mathrm{N} / \mathrm{A}$ & $\mathrm{N} / \mathrm{A}$ & $\begin{array}{c}\text { Before } \\
\mathrm{RT}=322 \\
\pm 116 \mathrm{~mL} ; \\
\text { After } \\
\mathrm{RT}=269 \pm 75 \\
\mathrm{~mL}\end{array}$ & $\mathrm{~N} / \mathrm{A}$ \\
\hline
\end{tabular}

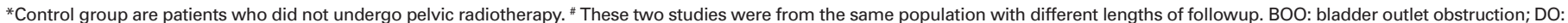

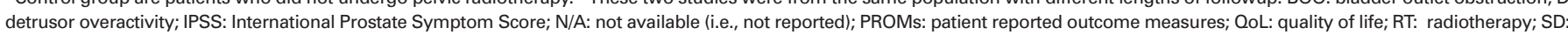
standard deviation; SEM: standard error of the mean; SRT: salvage radiotherapy; UDS: urodynamics study. 
de novo DO following radiotherapy. ${ }^{7,8}$ Another study with no baseline urodynamics reported rates of DO to be $37.5 \%$ at median of 7.7 years following radiotherapy. ${ }^{9}$

\section{Bladder compliance}

Only one study compared bladder compliance changes following radiotherapy to baseline. ${ }^{7,8}$ This study reported only a small percentage (12.5\%) of patients to have decreased bladder compliance at baseline. ${ }^{7,8}$ Overall, the percentage of patients with decreased compliance was only slightly worse at three months at $18.8 \% .{ }^{8}$ This progressively worsened with time, and at 18 months following radiotherapy, $33.3 \%$ of patients experienced a decreased bladder compliance. ${ }^{7}$ Ervandian et al did not compare bladder compliance with baseline but reported a high rate of impaired bladder compliance in $62.5 \%$ of men at median of 7.7 years following salvage radiotherapy. ${ }^{9}$ Mendez-Rubio et al compared the rate of decreased bladder compliance between patients who did and did not undergo pelvic radiotherapy and found pelvic radiotherapy to be statistically significantly predictor of decreased bladder compliance on univariate and multivariate analyses. ${ }^{10}$

\section{Bladder capacity}

Two studies compared mean maximum cystometric capacity (MCC) post-radiotherapy with baseline, ${ }^{7,8,11}$ and both reported a reduction in MCC following radiotherapy. One study reported a statistically significant reduction in mean MCC from $422.6 \mathrm{ml}$ to $352.9 \mathrm{ml}$ at three months, and further reduced to $328.6 \mathrm{ml}$ at 18 months. ${ }^{7,8}$ The same study also demonstrated bladder volume at first sensation and strong desire to be lower following radiotherapy at 18 months. ${ }^{7,8}$ Another study reported MCC reduced from $322 \mathrm{ml}$ to $269 \mathrm{ml}$ from 3-22 months following radiotherapy. ${ }^{11}$ Given there were only four patients in this pre-post analysis, statistical significance was not found..$^{11}$ This study involved patients who had adjuvant radiotherapy $(n=10)$ and compared the urodynamics findings with a group of patients who only had RP $(n=13)$ and found no difference in the MCC on followup. ${ }^{11}$ Mendez-Rubio et al compared patients who had pelvic radiotherapy with patients who did not and found mean bladder volume at first desire and MCC to both be statistically significantly worse compared to control. ${ }^{10}$ The mean MCC in radiotherapy patients was 175 $\mathrm{ml}$ compared to $236 \mathrm{ml}$ in the control group $(\mathrm{p}<0.001) .{ }^{10}$ Ervandian et al did not compare MCC with baseline, but at median of 7.7 years following salvage radiotherapy, the mean MCC appeared to be low at $297.8 \mathrm{ml}^{9}$

\section{Discussion}

This systematic review highlights the paucity of research in the current literature examining bladder function for patients following radiotherapy for localized prostate cancer. From the limited literature available, it appears that radiotherapy results in impaired bladder compliance and decreased bladder capacity. Resolution of BOO appears to be less frequent than in studies involving RP patients. ${ }^{12}$ De novo DO may develop following radiotherapy, especially with longer followup. As LUTS have a potentially significant impact on QoL, it is important to counsel patients adequately before consideration of curative treatment options for prostate cancer. The findings of this review emphasize the importance of adequate pre-treatment assessment of LUTS, in addition to post-treatment evaluation, to identify patients who may benefit from treatment to improve their QoL.

Bladder compliance appears to worsen following radiotherapy, ${ }^{7,8,10}$ especially with time. ${ }^{7,8}$ This is consistent with the mechanism of radiation-induced damage, which often manifests at a delayed time. ${ }^{13}$ Radiotherapy uses ionizing radiation to destroy tumor cells by increasing the production of free radicals and reactive oxygen species (ROS) that damage structural proteins and genetic material, ultimately leading to cell death. ${ }^{14}$ The effects of radiation are, however, not limited to malignant cells, causing collateral damage to surrounding healthy tissues.

Radiation-induced damage to vascular endothelial cells generates a reserve of long-lasting free radicals and ROS with subsequent inflammation, vascular hyperplasia, perivascular fibrosis, and end vascular occlusion. ${ }^{14}$ These pathological changes occur by 6-12 months following radiotherapy, but the resulting bladder fibrosis and degeneration of the bladder wall that occurs secondary to vascular ischemia of the bladder wall is generally seen months to years after radiotherapy.$^{13}$ This ultimately results in decreased bladder compliance and contraction. ${ }^{14}$ The high rate of poor bladder compliance seen in salvage radiotherapy is likely attributable more to radiotherapy than to RP, with a previous systematic review having shown that although bladder compliance is initially impaired following RP, this tends to recover with time. ${ }^{12,15}$

The mechanism for reduction in bladder capacity following radiotherapy, as demonstrated in the studies by Do et al, Choo et al, and Mendez-Rubio et al, is likely similar to that previously described for decreased bladder compliance. ${ }^{7,8,10}$ The study by Presti et al comparing RP and adjuvant radiotherapy with RP alone found no difference in MCC, but the numbers are likely too small to detect a difference. ${ }^{11}$ Furthermore, RP itself is a confounder, as it has been shown to reduce bladder compliance and contractility, which may be related to decentralization of the bladder from its mobilization during prostatectomy, bladder denervation due to disruption of branches of the pudendal nerve, postoperative inflammatory changes, and geometric bladder wall alteration. ${ }^{12}$ This effect of RP may recover over time, with Giannantoni et al showing an improvement in bladder compliance at 36 months following RP. ${ }^{15}$ There are no long- 
term studies to demonstrate a similar recovery in patients following radiotherapy. With the proposed mechanism secondary to fibrosis of the bladder, the reversibility of impaired bladder compliance and MCC following radiotherapy seems less likely. Further longer-term followup studies are required to elucidate this.

Prostate swelling caused by radiation is resolved in the majority of patients by three months, with only a small number experiencing de novo $\mathrm{BOO}$ that is ultimately resolved by 18 months. ${ }^{7,8}$ BOO does not appear to be worse at three and 18 months post-radiotherapy, with Qmax and PVR similarly unchanged. ${ }^{7,8}$ In contrast to a BOO improvement rate of $20-59.3 \%$ following $\mathrm{RP}^{12}{ }^{12}$ resolution of $\mathrm{BOO}$ post-radiotherapy is not as significant. Unlike RP, BPH can persist following radiotherapy. Clinically, this may be ameliorated by medical or surgical treatment of BOO before or after radiotherapy, as per usual indications for concurrent $\mathrm{BPH} .{ }^{16}$ The high rate of $\mathrm{BOO}$ reported by Ervandian et al following salvage radiotherapy is likely secondary to the combined effects of both RP and radiotherapy. ${ }^{9}$ The majority of patients in that cohort underwent open RP, which is known to be associated with a higher rate of vesicourethral anastomotic stenosis (VUAS) compared to robot-assisted RP. ${ }^{17}$ Furthermore, radiotherapy has been known to make VUAS worse and cause urethral stricture disease. ${ }^{18}$ As such, these patients may have a higher rate of BOO secondary to urethral stricture, and VUAS complications as a result of receiving both therapies.

Overactive bladder (OAB) symptoms appear to be more common and severe following radiotherapy than after RP. ${ }^{5}$ Similarly, radiotherapy does not result in the same degree of improvement of DO as seen following RP. ${ }^{12}$ DO appears to remain largely the same and possibly slightly worse at 18 months. ${ }^{7,8}$ Two possible explanations are thought to account for this. Firstly, radiotherapy may result in radiation cystitis, which is associated with lower urinary tract symptoms. ${ }^{14,19}$ It is plausible that with longer followup, the bladder effects secondary to radiation manifest themselves more prominently, as demonstrated in a study reporting the rate of DO following salvage radiotherapy to be as high as $37.5 \%$ at 7.7 years. ${ }^{9}$ Secondly, a significant portion of patients' DO at baseline is secondary to $\mathrm{BOO}$ from $\mathrm{BPH}$, and unlike RP, whereby $\mathrm{BOO}$ is relieved following removal of the prostate gland, radiotherapy does not resolve $\mathrm{BOO}$ to lead to the resolution of DO.

The systematic review is limited by the low level of evidence in the current literature and the scarce number of studies published in this area. The majority of studies had a small number of participants. Furthermore, incomplete reports of important parameters and outcomes measures examined in this review were common throughout studies. PROMs data on LUTS following radiotherapy was poorly reported in conjunction with urodynamic results. While studies have shown good correlation between symptoms and urodynamic findings, reported rates of $O A B$ symptoms may be higher than in urodynamic studies. ${ }^{20}$ Additionally, patients may also have urodynamic changes suggestive of $\mathrm{BOO}$ without clinical significance, due to the presence of a transurethral transducer obstructing flow and exacerbating the underlying condition. Future studies of urodynamics post-radiotherapy should, therefore, be performed in conjunction with PROMs. Finally, the absence of brachytherapy patient cohorts in the literature on this topic limits the extrapolation of the findings in this review to brachytherapy patients. Overall, given the current level of evidence available in the literature, it is difficult to draw any strong conclusions.

\section{Conclusions}

Radiotherapy for localized prostate cancer results in impaired bladder compliance and decreased bladder capacity, as demonstrated on urodynamics studies. Resolution of BOO appears less likely in comparison to case series on radical prostatectomy. De novo DO appears to develop following radiotherapy, especially with longer followup. With only low level of evidence studies available at present, further high-quality, prospective studies are important to elucidate the impact of radiotherapy on bladder function.

Competing interests: The authors do not report any competing personal or financial interests related to this work.

This paper has been peer-reviewed.

\section{References}

1. Ferlay J, Soerjomataram I, Dikshit R, et al. Cancer incidence and mortality worldwide: Sources, methods, and major patterns in GLOBOCAN 2012. Int J Cancer 2015;136:E359-86. https://doi.org/10.1002/ iic.29210

2. D'Amico AV, Whittington R, Malkowicz SB, et al. Biochemical outcome after radical prostatectomy, external beam radiation therapy, or interstitial radiation therapy for clinically localized prostate cancer. JAMA 1998;280:969-74. htrps://doi.org/10.1001/jama.280.11.969

3. Tewari A, Divine G, Chang P, et al. Long-term survival in men with high-grade prostate cancer: A comparison between conservative treatment, radiation therapy and radical prostatectomy — a propensity scoring approach. J Urol 2007;177:911-5. https://doi.org/10.1016/i.juro.2006.10.040

4. Eastham JA, Scardino PT, Kattan MW. Predicting an optimal outcome after radical prostatectomy: The trifecta nomogram. J Urol 2008;179:2207-10;discussion 2210-1. https://doi.org/10.1016/i. juro.2008.01.106

5. Thiruchelvam N, Cruz F, Kirby M, et al. A review of detrusor overactivity and the overactive bladder after radical prostate cancer treatment. BJU Int 2015;116:853-61. https://doi.org/10.1111/bju.13078

6. National Heart Lung and Blood Institute, Bethesda, MD. Study Quality Assessment Tools. Vol 2020. Available at: https://www.nhlbi.nih.gov/health-topics/study-quality-assessment-tools. Accessed June 22, 2021

7. Choo R, Do V, Herschorn S, et al. Urodynamic changes at 18 months post-therapy in patients treated with external beam radiotherapy for prostate carcinoma. Int J Radiat Oncol Biol Phys 2002;53:290-6. https://doi.org/10.1016/S0360-3016(02)02726-8 
8. Do V, Choo R, Deboer $G$, et al. Urodynamic findings 3 months after radiotherapy in patients treated with conformal external beam radiotherapy for prostate carcinoma. BJU Int 2002;90:62-7. https://doi.org/10.1046/i.1464-410X.2002.02813.x

9. Ervandian $M$, Diurhuus $J C$, Hoyer $M$, et al. Long-term urodynamic findings following radical prostatectomy and salvage radiotherapy. Scandinav J Urol 2018;52:20-6. https://doi.org/10.1080/21681805.2 017.1354067

10. Mendez-Rubio S, Salinas-Casado J, Virseda-Chamorro M, et al. Long-term adverse effects on bladder filling phase in males submitted to the pelvic radiotherapy. Arch Esp Urol 2015;68:609-14. https://pubmed. ncbi.nlm.nih.gov/26331397/

11. Presti JC, Roach M, Narayan PA, et al. Effect of adjuvant radiation therapy on urodynamic parameters following radical retropubic prostatectomy. Radiat Oncol Invest 1996;4:192-5. https://doi.org/10.1002/ (SICI) 1520-6823(1996)4:4<192::AID-ROI7>3.0.C0;2-T

12. Yao HH, Hoe V, Crump RT, et al. Impact of radical prostatectomy on bladder function as demonstrated on urodynamics study-A systematic review. Neurourol Urodyn 2021;40:582-603. https://doi.org/10.1002/nau.24606

13. Marks LB, Carroll PR, Dugan TC, et al. The response of the urinary bladder, urethra, and ureter to radiation and chemotherapy. Int I Radiat Oncol Biol Phys 1995;31:1257-80. https://doi.org/10.1016/03603016(94)00431-J

14. Geier M and Geinitz H. Late Toxicity and Quality of Life. In. Geinitz H, Roach III M, and van As N, editors. Radiotherapy in Prostate Cancer: Innovative Techniques and Current Controversies. Berlin, Heidelberg: Springer Berlin Heidelberg; 2015:75-86.
15. Giannantoni A, Mearini E, Zucchi A, et al. Bladder and urethral sphincter function after radical retropubic prostatectomy: a prospective long-term study. Eur Urol 2008;54:657-64. https://doi.org/10.1016/i. eururo.2007.10.054

16. Crawford $E D$ and Kavanagh $B D$. The role of alpha-blockers in the management of lower urinary tract symptoms in prostate cancer patients treated with radiation therapy. Am I Clin Oncol 2006;29:517-23. https://doi.org/10.1097/01.coc.0000225412.24750.4c

17. Breyer $B N$, Davis $C B$, Cowan JE, et al. Incidence of bladder neck contracture after robot-assisted laparoscopic and open radical prostatectomy. BJU Int 2010;106:1734-8. https://doi.org/10.1111/i.1464410X.2010.09333.X

18. Browne BM and Vanni AJ. Management of urethral stricture and bladder neck contracture following primary and salvage treatment of prostate cancer. Curr Urol Rep 2017;18:76. https://doi.org/10.1007/ s1 1934-017-0729-0

19. Goucher G, Saad F, Lukka H, et al. Canadian Urological Association best practice report: Diagnosis and management of radiation-induced hemorrhagic cystitis. Can Urol Assoc J 2019;13:15-23. https://doi.org/10.5489/cuaj.5788

20. Matharu G, Donaldson MM, McGrother CW, et al. Relationship between urinary symptoms reported in a postal questionnaire and urodynamic diagnosis. Neurourol Urodyn 2005;24:100-5. https://doi.org/10.1002/nau.20093

Correspondence: Dr. Henry Han-I Yao, Department of Surgery, University of Calgary, Calgary, AB, Canada; henry.yao@monash.edu

\begin{tabular}{|c|c|c|c|c|c|c|c|c|c|c|c|c|c|c|c|c|}
\hline \multicolumn{17}{|c|}{$\begin{array}{l}\text { Supplementary Table 1. Risk of bias assessments using the National Heart, Lung, and Blood Institute Study Quality } \\
\text { Assessment Tools }\end{array}$} \\
\hline Author & Q1 & Q2 & Q3 & Q4 & Q5 & Q6 & Q7 & $\mathbf{Q 8}$ & Q9 & Q10 & Q11 & Q12 & Q13 & Q14 & Rater 1 & Rater 2 \\
\hline Presti et al,1996"11* & Yes & No & $C D$ & $C D$ & No & Yes & Yes & Yes & Yes & Yes & Yes & $C D$ & No & No & Fair & Fair \\
\hline $\begin{array}{l}\text { Mendez-Rubio et al, } \\
2015^{10 *}\end{array}$ & Yes & No & $C D$ & $\mathrm{CD}$ & Yes & Yes & Yes & No & Yes & Yes & No & $\mathrm{CD}$ & $\mathrm{CD}$ & No & Fair & Fair \\
\hline Ervandian et al, $2018^{9 \#}$ & Yes & Yes & No & Yes & Yes & Yes & Yes & Yes & Yes & & & & & & Fair & Fair \\
\hline Do et al, $2002^{8 \wedge}$ & Yes & Yes & CD & No & No & Yes & Yes & $C D$ & Yes & Yes & Yes & NR & & & Good & Good \\
\hline Choo et al, $2002^{7 \wedge}$ & Yes & Yes & $\mathrm{CD}$ & No & No & Yes & Yes & $C D$ & Yes & Yes & Yes & NR & & & Good & Good \\
\hline
\end{tabular}

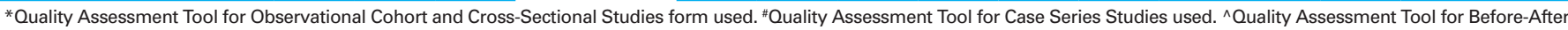
(Pre-Post) Studies with No Control Group used. CD: cannot determine; NR: not reported. 